A N N A L E S
UNIVERSITATIS MARIAE CURIE-SKŁODOWSKA L U B L I N - P OL ON I A

VOL. XXVIII, 10

SECTIO $\mathrm{H}$

1994

Zakład Organizacji i Zarządzanja Wydzialu Ekonomicznego UMcs

Zbigniew SZELOCH

\title{
Personalmanagement als Wissenschaftsdisziplin in Polen
}

Zarządzanie kadrami jako dyscyplina naukowa w Polsce

Personalmanagement tauchte als eigenständige wissenschaftliche Disziplin in Polen erst vor kurzem, und zwar am Anfang der neunziger Jahre, auf. Zwar wurden an einigen Hochschulen und Universitäten auch schon früher ähnlich genannte Vorlesungen zu diesem Fach gehalten, sie waren aber noch nicht allgemein verbreitet. Man darf dabei nicht vergessen, daß sich Hochschulen im System der zentral gelenkten Wirtschaft an Lehrpläne und Lehrprogramme des Zentrums - des Ministeriums für Hochschulwesen - halten mußten. Im Rahmen des zentral festgelegten Studienprogramms durften sich die Hochschulen nur einer beschränkten Autonomie erfreuen, die u.a. darauf beruhte, daß in die Studienprogramme die sòg. fakultativen Studienfächer aufgenommen werden konnten. Zu dieser Gruppe der Studienfächer gehörte auch die Problematik des Personalmanagements.

Das hat freilich nicht $\mathrm{zu}$ bedeuten, daß Personalmanagement im Rahmen der Vorlesungen, Seminare und Ubungen unterrichtet wurde. Personalmanagement war inhaltlicher Bestandteil solcher wissenschaftlicher Disziplinen wie Organisation und Führung, Organisationssoziologie, Führungssoziologie, Arbeitswirtschaftslehre, Psychologie der Führung, Beschäftigungspolitik, Arbeitspsychologie, Sozialpsychologie, die an Hochschulen und Universitäten unterrichtet wurden.

Darüber hinaus wurde Personalmanagement im Rahmen der Lehrveranstaltungen des Nachdiplomstudiums, der universitären Fachausbildung, der Lehrgänge und Schulungen, die durch sehr viele verschiedene Weiterbildungsinstitutionen und Schulungszentren durchgeführt wurden. Lehrgänge dieser Art waren vorwiegend kurzfristig (sie dauerten von einem Monat bis zu einem Jahr) und deren Ziel war Ergänzung und Aktualisierung des Wissens in diesem Bereich. 
Der im Jahre 1990 begonnene Prozeß der Systemumwandlung fand auch im Bereich der Fachausbildung seinen Niederschlag. Hochschulen und Universitäten bekamen eine große Autonomie sowohl in Bezug auf Studienrichtungen als auch in Bezug auf Lehrpläne und Studienprogramme.

Im Rahmen des wirtschaftswissenchaftlichen Studiums wurden vier Gruppen von Studienfächern festgelegt: gemeinsame, obligatorische Fächer, Fachausbildung und Spezialisierungsfächer. Die Studienfächer der ersten zwei Gruppen, durch das Ministerium für Volksbildung festgelegt, sind für Studenten und Hochschulen gleichermaßen Pflichtlehrveranstaltungen und sie bilden insgesamt zwei Drittel des allgemeinen Stundenpensums. Die Studienfächer der zwei übrigen Gruppen (d.i. die Fachausbildung und Spezialisierungsfächer) werden durch die Hochschulen selbständig festgelegt. Dabei werden im Rahmen der Fachausbildung in der Regel mehr - um ca. 50\% - fakultative Studienfächer zur freien Wahl der Studenten angeboten. Im Rahmen der Spezialisierungsfächer werden die sog. Spezialisierungsblocks zur Wahl angeboten.

Ich erwähne diese Gliederung aus dem Grunde, weil im Rahmen der Studienrichtung „Management und Marketing” in der Gruppe der obligatorischen Studienfächer auch das Studienfach „Personalmanagement" mit 30 Stunden Vorlesung im 6. Semester des zehnjährigen Hochschulstudiums ins Studienprogramm den Eingang gefunden hat.

Als zentral festgelegtes Pflichtfach wird es nur im Rahmen dieser Studienrichtung unterrichtet. Bei den anderen Studienrichtungen können die Hochschulen selbst Personalmanagement in die Studienprogramme aufnehmen, was sie auch gern tun.

Für das Studienfach „Personalmanagement” wurde das sogenannte Rahmenprogramm als Richtlinien für die Hochschullehrer und wissenschaftlich-didaktische Mitarbeiter entworfen. Das Rahmenprogramm besteht aus 6 Problemkreisen mit folgenden Programminhalten:

Problemkreis 1

Prämissen der Konstruktion eines Personalmanagementsmodells. Umweltbedingungen. Doktrinbedingungen. Wirtschaftlich-organisatorische Bedingungen. Die wichtigsten Merkmale des Modells. Inhaltlicher Umfang eines zeitgemäßen Modells.

Problemkreis 2

Personalmanagement im System der Unternehmensführung. Personalmanagement im Schrifttum. Struktur der Unternehmensführung. Struktur des Personalmanagements. Ziele des Personalmanagements. 


\section{Problemkreis 3}

Subjekte des Personalmanagements. Eigentum und Personalmanagement. Grundlagen der industriellen Verhältnisse. Ziele des Unternehmenseigners, des Personals und des Managements. Organe und Organisationseinheiten des Unternehmens.

\section{Problem kreis 4}

Verfahren und Techniken des Personalmanagements. Voraussetzungen für die Auswahl der Techniken. Personalplanung. Arbeitsstellenanalyse. Quellen und Verfahren der Personalgewinnung. Auswahl der Mitarbeiter. Orientierung und Anpassung der Mitarbeiter. Beurteilung der Mitarbeiter. Schulung der Mitarbeiter. Interpersonelle Kommunikation. Motivierung der Mitarbeiter. Entlassung.

\section{Problemkreis 5}

Organisation des Mitarbeiterdienstes. Funktionen und Aufgaben des Mitarbeiterdienstes. Gestaltung der Organisationsstruktur. Mitarbeiterprogramme. Kriterien der Beurteilung des Mitarbeiterdienstes.

Problemkreis 6

Kontrolle der Ziele des Personalmanagements. Zum Begriff der Kontrolle und deren Funktionen. Subjekte der Kontrolle. Informationssystem des Unternehmens und Kontrolle. Prozeß der Kontrolle. Kontrollbegleitende Maßnahmen.

In den anderen Studienrichtungen der Wirtschaftswissenschaftslehre wird die Problematik des Personalmanagements im Rahmen der Gruppe der Fachausbildung und der Spezialisierungsfächer unterrichtet, wobei die Bezeichnung dieser Fächer stark differenziert ist. So tragen sie u.a. folgende Bezeichnungen: Management des Menschenpotentials, Okonomie der Arbeit, Kaderpolitik, Menschenführung, Management der Arbeitsressourcen, Personalentwicklungsstrategie, Human Resources Management.

Zur Veranschaulichung dieses Problems werde ich im Folgenden Programmthesen von drei Fächern darstellen.

Das erste Fach ist „Personalpolitik” und wird im 3. Studienjahr der Wirtschaftswissenschaften im Rahmen des Spezialisierungsblocks „Management der Arbeitsressourcen" unterrichtet.

1. Gegenstand des Personalmanagements.

2. Modelle der Personalmanagements:
a) Traditionell,
b) Human Relations,
c) Human Resources. 
3. Koordinierungsverfahren im Rahmen des Human Resources Managements:

a) Strategische Planung und Management der Arbeitsressourcen,

b) Strategisches Management und Personalmanagement,

c) Konzeption der integrierten Unternehmensentwicklung.

4. Elemente der Personalpolitik.

5. Planung von Human Resources:

a) Planungsetappen,

b) Prognostizierung der Entwicklung der Human Resources.

6. Mitarbeitergewinnung:

a) Stellenbeschreibung,

b) Gewinnungsquellen.

7. Personalauswahl:

a) Auswahlstufen,

b) Befragung,

c) Auswahl der Manager,

d) Beurteilungsmittel.

8. Sozial-berufliche Anpassung.

9. Mitarbeiterbeurteilung.

10. Schulung und Personalentwicklung:

a) Schulungsprogramme,

b) Programme der Managerentwicklung.

11. Mitarbeiterbeförderung.

12. Personalführung und Leistung.

13. Leistungsbewertung:

a) Ziele,

b) Verfahren,

c) Universalverfahren zur Leistungsbewertung.

14. Rolle der Gewerkschaften bei der Personalpolitik des Unternehmens.

Das zweite Studienfach ist „Human Resources Management der Organisation" und wird im 3. Studienjahr der Wirtschaftswissenschaften im Rahmen des Spezialisierungsblocks „Management der Human Ressources” unterrrichtet.

1. Human Resources Management der Organisation

Personalfunktion: Grundsätze des Human Ressource Managements der Organisation, Entwicklungsrichtungen; Elemente des Pensonalmanagementsprozesses.

2. Personalplanung

Grundsätze der Personalplanung, Rolle, Verfahren zur Entwicklung der Personalplanung. 
3. Mitarbeitergewinnung

Mitarbeitergewinnungsaktion, allgemeine und Segmentgewinnung, ali ternative Möglichkeiten der Mitarbeitergewinnung.

4. A u s w a h 1

Verfahren und Techniken zur Durchführung der Auswahl, Auswahlkriterien und Organisationsstruktur.

5. Organis ationskultur

Organisationskultur als Ressourcen und Organisationspotential, Rolle der Personalfunktion bei der Entwicklung der Organisationskultur.

6. Personalbeurteilung

System der Personalbeurteilung.

7. Le is tung s b ew ertung

Arbeitsstellenanalyse.

8. Entlohnung

Lohnpolitik, Formen der Mitarbeiterentlohnung, Motivationsfunktion der Entlohnung.

9. Personalentwicklung

Persomalentwicklung und -förderung, Schulung als Investition; Verfahren zur Ermittlung der Investitionsrentabilität (Deckungsbeitragsrechnung, Kosten- und Vorteilanalyse).

10. Karriereplan ung

Planung der Mitarbeiterlaufbahn, alternative Förderungsmöglichkeiten, Erbschaftsbuch.

11. Industrial Relations - Relationen mit Gewerk$\mathrm{sch}$ af ten

12. Ethikundsoziale VerantwortlichkeitderOrga$n$ is ation und die Personalfunktion

Grundsätze der sozialen Verantwortlichkeit, ethisches Personalmanagement - Bedürfnis oder Notwendigkeit?

13. Soziales Berichtswesendes Unternehmens. Social Audit

Anfertigung des sozialen und personalen Berichtswesens des Unternehmens, Kontrollverfahren (audit); Elemente der Organisationsberatung im Bereich des Human Ressourcen Managements der Organisation.

14. Strategisches Human Resources Management der Organisation

Strategische Stufe des Personalmanagements im modernen Unternehmen, Strategie der Organisation und Personalmanagement.

15. Organisation der Personalfunktion

Organisation der Personalabteilung, zentrale bzw. dezentrale Organisation, Aufgaben der Personalabteilung. 
Das dritte Fach ist „Personalpolitik und -strategie” und wird im Rahmen des Nachdiplomstudiums unterrichtet.

1. Human Resources Management der Organisation

Personalfunktion; Grundsätze des Human Resources Managements, Entwicklungsrichtungen; Elemente des Persoalmanagementsprozesses.

2. Organisationstrategie und Personalmanagement

Rolle und Typen der Strategie, strategische Stufe des Personalmanagements im modernen Unternehmen, Organisationsstrategie und Personalmanagement.

3. Mitarbeitergewinnung und Auswahl

Mitarbeitergewinnungsaktion, allgemeine und Segmentgewinnung, alternative Möglichkeiten der Mitarbeitergewinnung, Verfahren und Techniken der Auswahl, Auswahlkriterien und Organisationsstrategie.

4. Person al be urteilung

System der Personalbeurteilung, Verfahren und Instrumente.

5. Entlohnung

Leistungsbewertung, Lohnpolitik, Formen der Personalentlohnung, Motivationsfunktion der Entlohnung.

6. Personalen twicklung

Personalschulung und -entwicklung, Schulung als Investition; Mitarbeiterförderung, alternative Laufbahnmöglichkeiten, Erbschaftsbuch.

Ich darf annnehmen, daß die angeführten Beispiele - drei Bespiele aus verschiedenen Hochschulen und ein Beispiel des sog. zentralen Rahmenprogramms - einen guten Einblick in die Problematik des Personalmanagements gewähren, wie es an den polnischen Hochschulen unterrichtet wird.

Die Analyse dieser Programme weist eindeutig darauf hin, daB die gegenwärtig realisierten Lehrveranstaltungen im Bereich des Personalmanagements in den vier nachfolgend genannten wissenschaftlichen Disziplinen ihre theoretische Fundierung haben. Es sind:

- Bewirtschaftung des Faktors Mensch,

- Organisations- und Führungstheorie,

- Organisationssoziologie,

- Psychologie des Managements.

Alle diese Disziplinen werden seit vielen Jahren im Rahmen des Studiums der Wirtschaftswissenschaften sowie der Soziologie unterrichtet.

Der Personalmanagement befindet sich in Polen in statu nascendi und wird - neben der Problematik aus den o.g. wissenschaftlichen Disziplinen - stark aus Westeuropa und USA beeinflußt. Der Ubergang von 
der zentral gelenkten Planwirtschaft zur Marktwirtschaft erfordert die Beschäftigung mit dieser Problematik, die in der Vergangenheit des „real existierenden Sozialismus" das Entscheidungsfeld mit vorwiegend politischem Charakter war.

Die Unternehmen, die dem wachsenden Wettbewerb Stirn bieten müssen, beginnen die Bedeutung der Mitarbeitergewinnung und des Personalmanagements $\mathrm{zu}$ begreifen und sie verlangen nach neuen Studienfächern und nach neuen, den veränderten Umweltbedingungen angepaßten Lösungen. Das alles schafft sehrr günstige Voraussetzungen für die Entwicklung dieser Disziplin, die - unabhängig von bestimmten allgemeingültigen Grundsätzen - die polnische Realität und die Umweltbedingungen mit geschichtlichem, soziologischem, rechtlichem und mentalem Hintergrund berücksichtigen muß. Verallgemeinerungen und Theorien, die unter anderen Bedingungen enstanden sind, lassen sich nicht vorbehaltlos automatisch und schematisch übertragen. Sie sind entsprechend der Realität anzupassen.

Die Untersuchungen, die ich unter den polnischen Wissenschaftlern durchgeführt habe, zeigten, daß die Bezeichnung dieser Disziplin .. „Personalmanagement" - in polnischer Sprache nicht adäquat ist und sogar, geschichtlich bedingt, eine leicht pejorative Aussage enthält. Viele Befragten neigten eher zur direkten Ubersetzung des Begriffes der englischen Sprache „Human Resources Management”, weil dies besser dem Geist der polnischen Sprache entspricht. Die Weiterentwicklung der Disziplin erblicken die Befragten in folgenden Bereichen:

- Soziale Aspekte der Änderungen in der Organisation (insb. im Zusammenhang mit den Eigentumsumwandlugen und der Umstrukturierung); firmen);

- Neue Formen der Mitarbeitergewinnung (über Arbeitsvermittler-

- Das Problem der Mitarbeiter- und Managerbeurteilung;

- Lohn als Motivationsfunktion;

- Moderne Techniken der Mitarbeiterführung;

- Organisationskultur und Organisationsklima;

- Strategisches Personalmanagement;

- Systembezogene (komplexe und interdisziplinäre) Erfassung der Personalfragen;

- Globalmanagement und interkulturelles Management;

- Integration normativer Lösungen mit den psychologischen und organisatorischen Verfahren und Techniken.

Die meisten Hochschulen in Polen realisieren wissenschaftliche Forschungsprojekte im Rahmen des Personalmanagements, die gegenwärtig folgenden Fragestellungen gelten: 
- Organisationskultur,

- Leistungsmotivation unter den Bedingungen der entstehenden Marktwirtschaft,

- Arbeitsmarkt und sein Einfluß auf die Tätigkeit der Unternehmen;

- Veränderungen in der Personal- und Lohnpolitik im Prozeß der Systemumwandlung;

- Arbeitslosigkeit und ihre sozialen Auswirkungen;

- Beschäftigung der Behinderten;

- Qualifizierung der Mitarbeiter;

- Produktivität;

- Förderung der Führungskräfte;

- Techniken der Mitarbeiterführung;

- Arbeitsethik.

Bei der Realisierung dieser Forschungsprojekte stößt man auf die Barriere der Knappheit an Finanzmitteln. Eine Lösung dieses Problems ist die Zusammenarbeit mit ausländischen Universitäten und Hochschulen, insbesondere aus Westeuropa, im Rahmen der Zusammenarbeitsverträge sowie der Programme Tessa und Phare.

Vom Standpunkt der Didaktik ist der Mangel an geeigneten Lehrbüchern ein großes Hindernis bei der Entwicklung dieser Disziplin. Es sinu in letzter Zeit zwar einige Arbeiten erschienen, die allendings nur einige Aspekte des Personalmanagements behandeln und einen eher populärwissenschaftlichen Charakter haben.

Zur Veranschaulichung des Gesamtbildes der Problematik des Personalmanagements muß man sagen, daß es an über hundert Schulungszentren und Weiterbildungszentren unterrichtet wird, die in den Jahren 1990-1993 wie Pilze nach dem Regen geschossen sind. Diese Schulungszentren haben jeweils einen eigenen Namen: Dazu gehören BusinessSchulen, Managerschulen, Managerweiterbildungszentren etc. Es sind private, staatliche und gesellschaftliche und gemischte Bildungsanstalten, die in differenzierten organisatorisch-rechtlichen Formen funktionieren. Sie entwickeln sich sehr dynamisch und zeichnen sich allgemein durch pragmatischen Ansatz und schnelles Reaktionsvermögen auf die Bedürfnisse der Nationalwirtschaft aus. Viele der genannten Schulungszentren arbeiten mit ähnlichen Instituten in Westeuropa und in den USA zusammen. Häufig werden Referenten aus dem Ausland herangezogen.

Auf Grund dieser Beziehungen sind diese Schulungszentren sehr schnell westeuropäische und amerikanische Lösungen an die polnischen Umweltbedingungen anzupassen. So werden auch Sonderlehrgänge im Bereich des Personalmanagements organisiert.

Im Folgenden stelle ich zwei Beispiele von Seminarprogrammen aus dem Bereich des Personalmanagements dar: 
Das Studium „Human Resources im Unternehmen”:

Personalmanagement und Mitarbeiterauswahl, Maslows Bedürfnispyramide, Faktoren der Menschenmotivation, Führungsstile, Manager und sein leadership, Manager und sein Team, Methoden bei der Mitarbeiterauswahl, Konflikte und Konfliktlösungen, praktische Unternehmensführung.

Das Studium des Personalmanagers:

1. Organisationsentwicklung und -klima. Rolle des Personalmanagers. Selbstentwicklung - Selbstdiagnose. Prognostizierung der Selbstentwicklung. Problemlösungsverfahren, Zusammenarbeit mit und im Team. Strategisches Personalmanagement.

2. Die Kunst der Verständigung. Stress am Arbeitsplatz. Verständigung mit den Vorgesetzten, den Mitarbeitern und den Kunden. Führung in Konfliktsituationen. Selbstdarstellung. Öffentliches Auftreten.

3. Gewinnung und Auswahl der Führungskräfte. Formulierung der Qualifikationsforderungen. Dokumentenanalyse. Vorstellungsgespräch. Managerstellenausschreibung. Mitarbeitergewinnung über Personalberatungsfirmen.

4. Mitarbeitermotivation und -förderung. Anreize des Motivationspotentials. Teamarbeit. Materielle und nichtmaterielle Motivation. Belohnung und Bestrafung. Entwicklung des Entlohnungssystems. Planung der Berufslaufbahn.

5. Personalführung. Selbstentwicklung des Mitarbeiters und die Unternehmensentwicklung. Verfahren und Techniken zur Beurteilung der Mitarbeiter. Führungsstile.

6. Verhandlungen. Konfliktlösungsverfahren.

7. Arbeistrecht.

8. Personalmanager - Verursacher der Veränderungen.

Ich möchte zusammenfassend festellen, daß sich die neue Wissenschaftsdisziplin in Polen kraft eigener Erfahrungen und unterstützt durch das Ausland sehr dynamisch entwickelt. Es stehen uns genug Wissenschaftler zur Verfügung, die sich mit dieser Problematik beschäftigen. Deren Bemühungen sollten nur durch entsprechende wissenschaftliche Literatur (Bücher, Fachzeitschriften, die mangels Finanzmittel nicht zur Verfügung stehen), internationale Kontakte und durch Finanzmittel für empirische Untersuchungen und für ausländische Forschungsaufenthalte, insb. für junge wissenschaftliche Mitarbeiter, unterstützt werden. 


\section{S T R E S Z C Z E I E}

Zarządzenie personelem, jako wyodrębniona dyscyplina naukowa, pojawiło się w Polsce stosunkowo niedawno, na początku lat 90. Natomiast problematyka tej dyscypliny znajdowała już przedtem swe odbicie $w$ takich przedmiotach, jak teoria zarządzania, socjologia zarządzania, ekonomika pracy, psychologia zarządzania i inne.

Aktualnie przedmiot ten wykładany jest na wszystkich uczelniach ekonomicznych i na wszystkich kierunkach studiów ekonomicznych z tym, że jego nazwa jest zróżnicowana (zarządzanie kadrami, polityka osobowa, zarządzanie potencjałem spolecznym, polityka personalna, gospodarka czynnikiem ludzkim).

Większość naukowców skłania się do przyjęcia nazwy „Zarządzanie potencjalem spolecznym", co odpowiadaloby angielskiej nazwie Human Resource Management, stosowanej powszechnie zarówno w Stanach Zjednoczonych AP, jak i w niektórych krajach Europy zachodniej.

Przeprowadzone przeze mnie w roku 1993 badania wykazuja, że dalszy kierunek rozwoju tej dyscypliny widzą polscy naukowcy szczególnie w następujących obszarach:

- społeczne aspekty zmian w organizacji (zwłaszcza w związku ze zmianami własnościowymi i restrukturyzacją);

- nowe formy zatrudnienia (poprzez wyspecjalizowane firmy);

- problematyka ocen pracowników i kierowników;

- motywacyjna funkcja płacy;

- nowoczesne techniki kierowania zespołami ludzkimi;

- kuitura organizacyjna i klimat organizacyjny,

- zarządzanie strategiczne potencjałem społecznym,

- systemowe (a więc kompleksowe i interdyscyplinarne) ujmowanie zagadnień personalnych,

- zarządzanie globalne i interkulturowość,

- integracja rozwiązań normatywnych z metodami i technikami psychologicznymi i organizatorskimi;

Ta nowa w naszych warunkach dyscyplina naukowa rozwija się w Polsce bardzo dynamicznie na podstawie własnych doświadczeń oraz wspólpracy międzynarodowej. Istnieje wystarczająca liczba naukowców zajmujących się tą problematyką. Należaloby tylko wesprzec ich odporwiednią literaturą (książki i specjalistyczne czasopisma) poglębionymi kontaktami międzynarodowymi oraz środkami na badania empiryczne i studia zagraniczne, szczególnie dla mlodych adeptów tej dyscypliny. 